UDC 66.011/66.012:662.765

\title{
SOLUTION OF THE PROBLEMS OF NON-STATIONARITY PROCESSES IN ETHYLENE REGION OF A CHEMICAL TECHNOLOGICAL COMPLEX ON PROCESSING THE CRACKING AND PYROLYSIS GASES
}

\author{
I.I.Osmanova \\ M.Nagiyev Institute of Catalysis and Inorganic Chemistry \\ agil-s@mail.ru \\ Received 14.03.2019
}

\begin{abstract}
Dynamics of the processes in the greatest - ethylene region of chemical-technological complex has been studied, the functions taking into account non-stationarity of the processes has been chosen, their dynamic kinetic model were constructed, and on the basis of entire mathematical descriptions there have been decided the tasks of their optimal control. This in result enabled to fulfill the goal to be achieved to maintain the optimum production capacity of the target products found out at optimization of chemical-technological complex for stationary conditions of their proceeding.
\end{abstract}

Keywords: non-stationarity, functions of deactivation, of control, productivity capacity, dynamics, catalyst, activity, block-flowsheet, cracking and pyrolysis gases.

\section{https://doi.org/10.32737/0005-2531-2019-3-75-83}

\section{Introduction}

To answer the Republics demands olefins obtained from cracking and pyrolysis gases from BNZ named after H.Aliyev and equipment EP300 of Sumgait plant Ethylene-Polyethylene and their rational using in the Institute of Catalysis and Inorganic Chemistry, named after M.Nagiev, Azerbaijan National Academy of Sciences there have been developed the chemical-technological complex (CTC) on co-processing of these gases. Functioning of such CTC will permit to achieve the needed productivity capacity of the target products, the Republic demands, will minimum expenditures Calculation of the processes entering CTC has been made by mathematical models constructed for stationary conditions of their proceeding. However, the processes considered in CTC, both catalytic and non-catalytical, often take place in conditions of non-stationarity caused by various reasons [7]. Depending on conclusions of running the processes, change in their activity in time occurs differently decreasing reactors production activity, term of catalysts operation, selectivity of the process.

Our task consists in that data on optimal productivities capacity of the target products obtained at optimization of an entire CTC and meeting the Republics need in them, woaldut be destroyed because of possible non-stationarity of the processes. This can be provided by introducing into the kinetic models of stationary processes the corresponding functions of non-stationarity, and on their basis to operate them by the entire mathematical models of the processes, each taken separately.

\section{Fulfillment of work}

Based on work done the article offers the flow sheet of these basic actions which were implemented for each process of the region for obtaining demanded results, namely:

1. design of a stoichiometric flowsheet of the process on the basis of experimental data;

2. working out the kinetic models of the process on the basis of stoichiometric flow sheet;

3. making up complete mathematical model of the process for stationary conditions on the basis of kinetic model and studying heat and hydrodynamic conditions in a reactor;

4. choice of the functions of process nonstationarity based on studying its reasons;

5. receiving dynamic mathematical model of the process;

6. working out algorithm of operating the process using non-stationary mathematical description;

7. obtaining results of operating the process in non-stationary conditions.

Below there are cited the results of the successive fulfillment of suggested scheme for the concrete processes of ethylene region in CTC. The regime parameters of the process and designations to them are offered in references corresponding to each process. 


\section{Pyrolysis of ethane [1, 2]}

1. Stoichiometric scheme of the process

$$
\begin{aligned}
& \mathrm{C}_{2} \mathrm{H}_{6} \stackrel{k_{1}}{\longrightarrow} \mathrm{C}_{2} \mathrm{H}_{4}+\mathrm{H}_{2} \\
& \mathrm{C}_{2} \mathrm{H}_{4}+2 \mathrm{H}_{2} \stackrel{k_{2}}{\longrightarrow} 2 \mathrm{CH}_{4} \\
& \mathrm{C}_{2} \mathrm{H}_{4} \stackrel{k_{3}}{\longrightarrow} \frac{1}{4} \mathrm{C}_{4} \mathrm{H}_{6}+\frac{1}{8} \mathrm{C}_{4} \mathrm{H}_{8}+\frac{1}{8} \mathrm{C}_{4} \mathrm{H}_{10}+\frac{1}{8} \mathrm{H}_{2} \\
& \mathrm{C}_{2} \mathrm{H}_{4} \stackrel{k_{4}}{\longrightarrow} \frac{1}{3} \mathrm{C}_{6} \mathrm{H}_{6}+\mathrm{H}_{2} \\
& \mathrm{C}_{2} \mathrm{H}_{4} \stackrel{k_{5}}{\longrightarrow} \mathrm{C}_{2} \mathrm{H}_{2}+\mathrm{H}_{2} \\
& \mathrm{C}_{2} \mathrm{H}_{4} \stackrel{k_{6}}{\longrightarrow} 2 \mathrm{C}+2 \mathrm{H}_{2} \\
& \mathrm{C}_{2} \mathrm{H}_{4}+\mathrm{C}_{2} \mathrm{H}_{6} \stackrel{k_{7}}{\longrightarrow} \frac{143}{150} \mathrm{C}_{3} \mathrm{H}_{6}+\frac{57}{150} \mathrm{C}_{3} \mathrm{H}_{8}+\frac{93}{150} \mathrm{H}_{2} \\
& \mathrm{C}+\mathrm{H}_{2} \mathrm{O} \stackrel{k_{8}}{\longrightarrow} \mathrm{CO}+\mathrm{H}_{2} \\
& \mathrm{CO}+\mathrm{H}_{2} \mathrm{O} \stackrel{k_{9}}{\longrightarrow} \mathrm{CO}_{2}+\mathrm{H}_{2}
\end{aligned}
$$

2. Stationary kinetic model

$$
\begin{aligned}
& \frac{\mathrm{d} n_{\mathrm{C}_{2} \mathrm{H}_{6}}}{\mathrm{~d} l}=-u^{-1}\left[k_{1} n_{\mathrm{C}_{2} \mathrm{H}_{6}}+k_{7} n_{\mathrm{C}_{2} \mathrm{H}_{6}} n_{\mathrm{C}_{2} \mathrm{H}_{4}} P / R T \sum_{i} n_{i}-\left(k_{1} / K_{p 1}\right) n_{\mathrm{C}_{2} \mathrm{H}_{4}} n_{\mathrm{H}_{2}} P / R T \sum_{i} n_{i}\right], \\
& \frac{\mathrm{d} n_{\mathrm{C}_{2} \mathrm{H}_{4}}}{\mathrm{~d} l}=u^{-1} n_{\mathrm{C}_{2} \mathrm{H}_{4}}\left\{k_{1} n_{\mathrm{C}_{2} \mathrm{H}_{6}} / n_{\mathrm{C}_{2} \mathrm{H}_{4}}-\left(k_{1} / K_{p 1}\right) n_{\mathrm{H}_{2}} P / R T \sum_{i} n_{i}-k_{2}\left[n_{\mathrm{H}_{2}} P / R T \sum_{i} n_{\mathrm{i}}\right]^{2}+\left(k_{1} / K_{p 2}\right) \times\right. \\
& \left.\times\left(n_{\mathrm{C}_{2} \mathrm{H}_{4}}^{2} / n_{\mathrm{C}_{2} \mathrm{H}_{4}}\right) P / R T \sum_{i} n_{i}-k_{3}-k_{4}-k_{5}-k_{6}-k_{7} n_{\mathrm{C}_{2} \mathrm{H}_{6}} P / R T \sum_{i} n_{i}\right\}, \\
& \frac{\mathrm{d} n_{\mathrm{H}_{2}}}{\mathrm{~d} l}=u^{-1}\left[k_{1} n_{\mathrm{C}_{2} \mathrm{H}_{6}}+\left(k_{2} / K_{p 2}\right) n_{\mathrm{C}_{2} \mathrm{H}_{4}}^{2} P / R T \sum_{i} n_{i}+n_{\mathrm{C}_{2} \mathrm{H}_{4}}\left[-\left(k_{1} / K_{p 1}\right) n_{\mathrm{H}_{2}} P / R T \sum_{i} n_{i}-\right.\right. \\
& \left.-2 k_{2}\left(n_{\mathrm{H}_{2}} P / R T \sum_{i} n_{i}\right)^{2}+1 / 8 k_{3}+k_{4}+k_{5}+2 k_{6}+(93 / 150) k_{7} n_{\mathrm{C}_{2} \mathrm{H}_{6}} P / R T \sum_{i} n_{i}\right]+ \\
& \left.+n_{\mathrm{H}_{2} \mathrm{O}} P\left(k_{8} n_{\mathrm{C}}+k_{9} n_{\mathrm{CO}}\right) / R T \sum_{i} n_{i}\right], \\
& \frac{\mathrm{d} n_{\mathrm{CH}_{4}}}{\mathrm{~d} l}=2 k_{2} \mathrm{u}^{-1}\left[n_{\mathrm{C}_{2} \mathrm{H}_{4}}\left(n_{\mathrm{H}_{2}} P / R T \sum_{i} n_{i}\right)^{2}-\left(1 / \mathrm{K}_{\mathrm{p} 2}\left(n_{\mathrm{CH}_{4}} P / R T \sum_{i} n_{i}\right)^{2}\right],\right. \\
& \frac{\mathrm{d} n_{\mathrm{C}_{4} \mathrm{H}_{6}}}{\mathrm{~d} l}=(1 / 4) u^{-1} k_{3} n_{\mathrm{C}_{2} \mathrm{H}_{4}}, \frac{\mathrm{d} n_{\mathrm{C}_{4} \mathrm{H}_{8}}}{\mathrm{~d} l}=(1 / 8) u^{-1} k_{3} n_{\mathrm{C}_{2} \mathrm{H}_{4}}, \frac{\mathrm{d} n_{\mathrm{C}_{4} \mathrm{H}_{10}}=(1 / 8) u^{-1} k_{3} n_{\mathrm{C}_{2} \mathrm{H}_{4}}, \mathrm{~d} n_{\mathrm{C}_{6} \mathrm{H}_{6}} / \mathrm{d} l=(1 / 3) u^{-1} k_{4} n_{\mathrm{C}_{2} \mathrm{H}_{4}},}{\mathrm{~d}},
\end{aligned}
$$




$$
\begin{aligned}
& \mathrm{d} n_{\mathrm{C}_{2} \mathrm{H}_{2}} / \mathrm{d} l=u^{-1} k_{5} n_{\mathrm{C}_{2} \mathrm{H}_{4}}, \mathrm{~d} n_{\mathrm{C}} / \mathrm{d} l=u^{-1}\left(2 k_{6} n_{\mathrm{C}_{2} \mathrm{H}_{4}}-k_{8} n_{\mathrm{C}} n_{\mathrm{H}_{2} \mathrm{O}} P / R T \sum_{i} n_{i}\right), \\
& d \mathrm{n}_{\mathrm{C}_{3} \mathrm{H}_{6}} / \mathrm{d} l=(143 / 150) u^{-1} k_{7} n_{\mathrm{C}_{2} \mathrm{H}_{4}} n_{\mathrm{C}_{2} \mathrm{H}_{6}} P / R T \sum_{i} n_{i}, \\
& \mathrm{~d} n_{\mathrm{C}_{3} \mathrm{H}_{8}} / \mathrm{d} l=(57 / 150) u^{-1} k_{7} n_{\mathrm{C}_{2} \mathrm{H}_{4}} n_{\mathrm{C}_{2} \mathrm{H}_{6}} P / R T \sum_{i} n_{i}, \\
& \mathrm{~d} n_{\mathrm{CO}} / \mathrm{d} l=u^{-1} n_{\mathrm{H}_{2} \mathrm{O}} P\left(k_{8} n_{\mathrm{C}}-k_{9} n_{\mathrm{CO}}\right) / R T \sum_{i} n_{i}, \\
& \mathrm{~d} n_{\mathrm{CO}_{2}} / \mathrm{d} l=u^{-1} k_{9} n_{\mathrm{CO}} n_{\mathrm{H}_{2} \mathrm{O}} P / R T \sum_{i} n_{i} .
\end{aligned}
$$

3. Equation of heat balance and hydrodynamics $g_{\mathrm{C}_{2} \mathrm{H}_{4}}^{\text {nont }} \sim g_{\mathrm{C}_{2} \mathrm{H}_{4}}^{\text {nont }}$

$$
\frac{\mathrm{d} T}{\mathrm{~d} L}=\frac{\pi d_{\mathrm{H}} q-\sum_{j} r_{j} \Delta H_{R j}}{\sum_{i}\left(\frac{g_{\mathrm{C}_{2} \mathrm{H}_{6}}^{0}}{\overline{\mathrm{M}}} \sum_{i} \alpha_{i}^{\prime}+\sum_{i} \Delta n_{i}\right) C_{p i}}
$$

$$
\frac{\mathrm{d} P}{\mathrm{~d} L}=-2160.4 \cdot 10^{-13}\left(1+\frac{30 d_{\mathrm{B}}}{L_{0}}\right) \frac{g_{\mathrm{C}_{2} \mathrm{H}_{6}}^{0}}{P_{0} d_{\mathrm{ins}}^{5}} .
$$

4. Account of the process non-stationaryity

Equation of cokeformation rate:

$$
r_{\mathrm{C}}=\frac{\mathrm{d} C}{\mathrm{~d} \tau}=k_{1} \cdot C_{4}^{+}=k_{01} \exp \left(-\frac{E_{1}}{R T}\right) \cdot C_{4}^{+}
$$

increase in coke thickness for time break $\Delta \tau$ :

$$
\Delta t_{\mathrm{c}}=\frac{r_{\mathrm{C}} \Delta \tau}{\rho_{\mathrm{C}}}
$$

change of pipe diameter as a result of coke deposit:

$$
\Delta d_{\mathrm{ins}}=d_{\mathrm{ins}}^{0}-d_{\mathrm{ins}}^{f}=2 \cdot \Delta t_{\mathrm{C}}
$$

5. Equation of pressure loss in dynamic conditions:

$$
\frac{\mathrm{d} P}{\mathrm{~d} L}=-2160.4 \cdot 10^{-13}\left(1+\frac{30 d_{\mathrm{ins}}^{\mathrm{f}}(\tau)}{L_{0}}\right) \frac{g_{\mathrm{C}_{2} \mathrm{H}_{6}}^{0}}{P_{0}\left(d_{\mathrm{ins}}^{f}(\tau)\right)^{5}} .
$$


6. Function of control:

- regressive dependences of ethylene yield on time of stove work $\tau\left(\mathrm{X}_{1}\right)$, reactor leading $g_{\mathrm{C}_{2} \mathrm{H}_{6}}^{0}\left(X_{2}\right)$ and change of pressure in it $\Delta P\left(X_{3}\right)$ :

$Y_{\text {cal }}=0.1094 X_{1}+0.4853 X_{2}-316.1281 X_{3}$
$\bar{Y}_{\mathrm{c} a l}=-5646+21.24 Y_{\mathrm{c} a l}-0.02295 Y_{\mathrm{cal}}^{2}+$ $+8.256 \cdot 10^{-6} Y_{\text {cal }}^{3}$.

The system of equations (2), (3), (8) with account of (9), (10) is a complete mathematical model of the process for non-stationary conditions.

Block-scheme of process operation in non-stationary conditions:

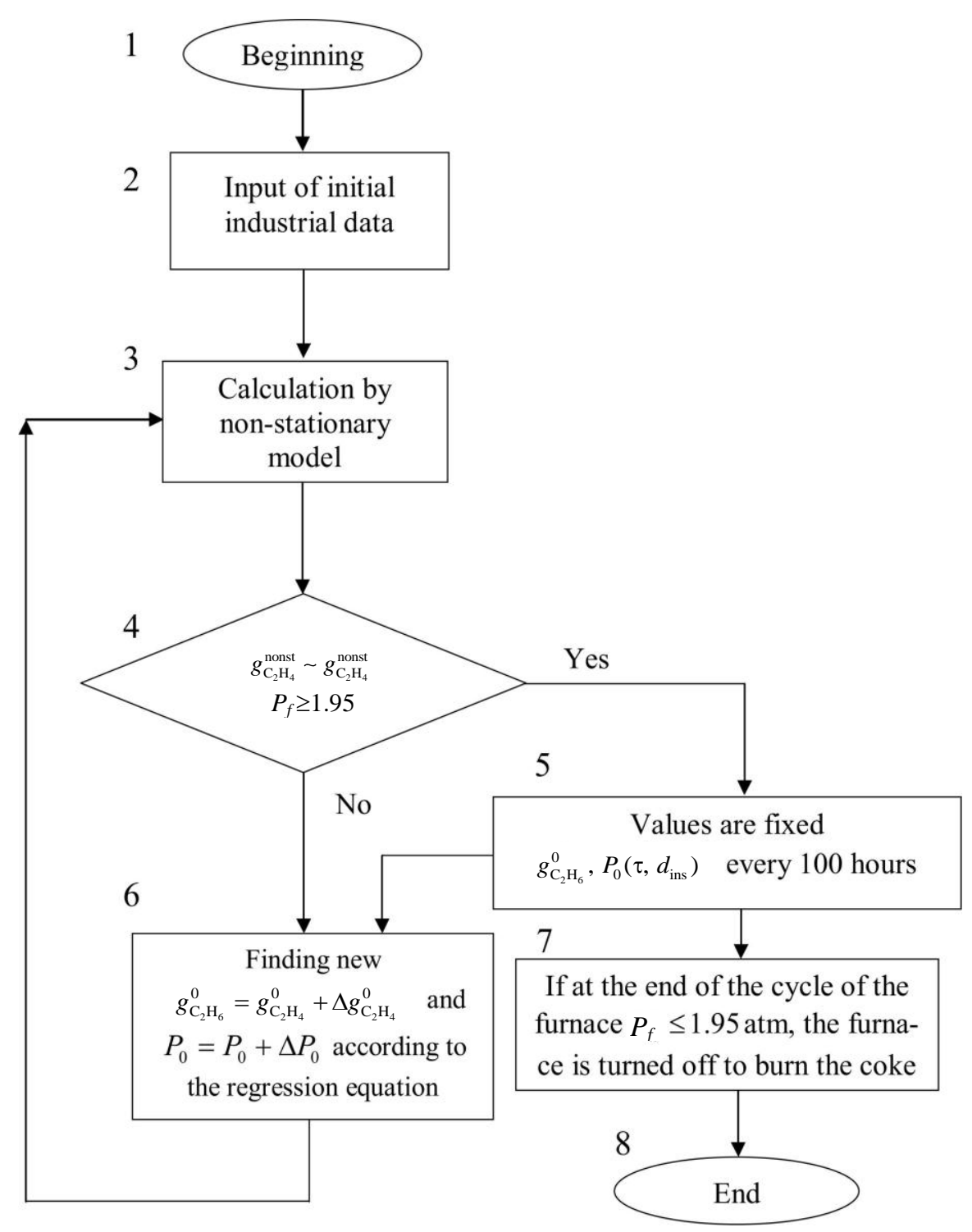


7. Results of calculation:

Table 1. The course and results of calculation of non-stationary process of ethane pyrolysis

\begin{tabular}{|c|c|c|c|c|c|c|c|c|c|c|c|c|}
\hline 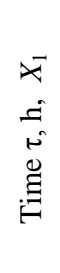 & $\begin{array}{c}\text { Load of } \\
\text { reactor } \\
g_{\mathrm{C}_{2} \mathrm{H}_{6}}^{0} \\
\mathrm{\kappa g} / \mathrm{h} \\
X_{2}\end{array}$ & 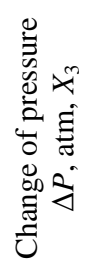 & 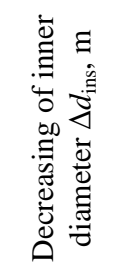 & $\begin{array}{c}\text { Final } \\
\text { diame- } \\
\text { ter, } \\
d_{\text {ins }}^{f}, \\
\mathrm{~m}\end{array}$ & 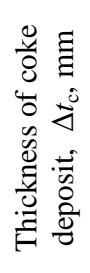 & 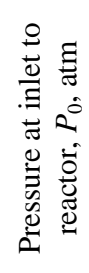 & 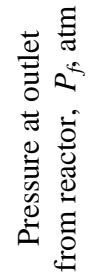 & $\begin{array}{c}\text { Yield of } \\
\text { ethylene } \\
g_{\mathrm{C}_{2} \mathrm{H}_{4}}^{\text {exs }} \\
Y_{\text {exs }}\end{array}$ & $\begin{array}{c}\text { Yield of } \\
\text { ethylene } \\
g_{\mathrm{C}_{2} \mathrm{H}_{4}}^{\mathrm{cal}} \\
Y_{\text {cal }}^{(5)}\end{array}$ & $\begin{array}{l}\text { Relative } \\
\text { error } \\
\frac{\left(Y_{\text {exs }}-Y_{\text {cal }}^{(5)}\right)}{Y_{\text {exs }}} \cdot 100 \%\end{array}$ & $\begin{array}{c}\begin{array}{l}\text { Yield of } \\
\text { ethylene }\end{array} \\
g_{\mathrm{C}_{2} \mathrm{H}_{4}}^{\text {cal }} \\
\overline{\mathrm{Y}}_{\mathrm{cal}}^{(6)}\end{array}$ & $\begin{array}{l}\text { Relative error } \\
\frac{\left(Y_{\mathrm{exs}}-\overline{\mathrm{Y}}_{\mathrm{cal}}^{(6)}\right)}{\mathrm{Y}_{\mathrm{exs}}} \cdot 100 \%\end{array}$ \\
\hline 100 & 2504 & 0.904 & 0.00424 & 0.1280 & 2.12 & 3.245 & 2.341 & 900.14 & 940.35 & -4.46 & 898.3 & 0.2073 \\
\hline 200 & 2510 & 1.088 & 0.00898 & 0.1232 & 4.50 & 3.253 & 2.165 & 901.1 & 896.04 & 0.56 & 899.1 & 0.217 \\
\hline 300 & 2526.8 & 1.252 & 0.01274 & 0.1195 & 6.40 & 3.274 & 2.022 & 899.5 & 863.28 & 4.02 & 898.1 & 0.1536 \\
\hline 400 & 2619.3 & 1.438 & 0.01605 & 0.1162 & 8.02 & 3.395 & 1.958 & 900 & 860.31 & 4.41 & 897.9 & 0.235 \\
\hline 500 & 2993.8 & 1.922 & 0.02030 & 0.1119 & 10.20 & 3.878 & 1.956 & 901.13 & 899.99 & 0.12 & 899.1 & 0.2226 \\
\hline 600 & 3057.6 & 2.018 & 0.02410 & 0.1081 & 12.05 & 3.970 & 1.952 & 900.46 & 911.55 & -1.23 & 899 & 0.1669 \\
\hline 700 & 3086.4 & 2.05 & 0.02950 & 0.1027 & 14.75 & 4.000 & 1.950 & 900.61 & 926.35 & -2.86 & 898.6 & 0.2222 \\
\hline
\end{tabular}

Yield of ethylene is maintained at demanded stationary level $(\approx 900 \mathrm{~kg} / \mathrm{h})$ and limit for end pressure is not destroyed.

\section{Ethylene polymerization [3]}

1. Stoichiometric scheme of the process

$$
n \mathrm{~A} \rightarrow(\mathrm{A})_{n}
$$

2. Stationary kinetic model:

$$
\begin{aligned}
& \frac{\mathrm{d} x}{\mathrm{~d} l}=\left[-\mathrm{A}_{\mathrm{R}}[\mathrm{M}]_{0} \exp \left(-\frac{b_{1}}{T}\right) \cdot T y^{1 / 2}\right], \\
& \frac{\mathrm{d} y}{\mathrm{~d} l}=-\mathrm{A}_{\mathrm{I}}[\mathrm{M}]_{0} \exp \left(-\frac{\mathrm{b}_{2}}{\mathrm{~T}}\right) y .
\end{aligned}
$$

3. Equation of heat balance

$$
\begin{aligned}
& \frac{\mathrm{d} T}{\mathrm{~d} l}=-a_{3} \exp \left(-\frac{b_{1}}{T}\right) x y^{1 / 2}+a_{4}\left(T-T_{0}\right), \\
x & =[\mathrm{M}] /[\mathrm{M}]_{0}, \\
y & =[I] /[\mathrm{M}]_{0},
\end{aligned}
$$

$$
\begin{aligned}
& b_{1}=-\left(E_{\mathrm{R}}+\Delta V \Delta p\right) / \mathrm{R}, \\
& b_{2}=E_{\mathrm{I}} / \mathrm{R} ; \\
& a_{3}=\mathrm{A}_{\mathrm{R}}[\mathrm{M}]_{0} Q_{R} ; \\
& a_{4}=-4 K / \pi d_{\text {ins }}^{2} C_{p} \rho
\end{aligned}
$$

\section{Function of non-stationarity}

$$
\theta=\mathrm{e}^{-\left(\mathrm{Ae}^{-\frac{E_{\mathrm{in}}}{R T}}\right) t_{\mathrm{R}} \cdot b \cdot F_{\mathrm{C}_{2} \mathrm{H}_{4}}}
$$

5. Dynamic kinetic model (equations (16) and (13))

$\frac{\mathrm{d} x}{\mathrm{~d} l}=\left[-\mathrm{A}_{\mathrm{R}}[\mathrm{M}]_{0} \exp \left(-\frac{b_{1}}{T}\right) \cdot T y^{1 / 2}\right] \cdot \theta$.

The system of equation (16), (13), (14) with account of (15) is a complete kinetic model of the process for non-stationary conditions. 
6. Block-scheme of process operating in non-stationary conditions:

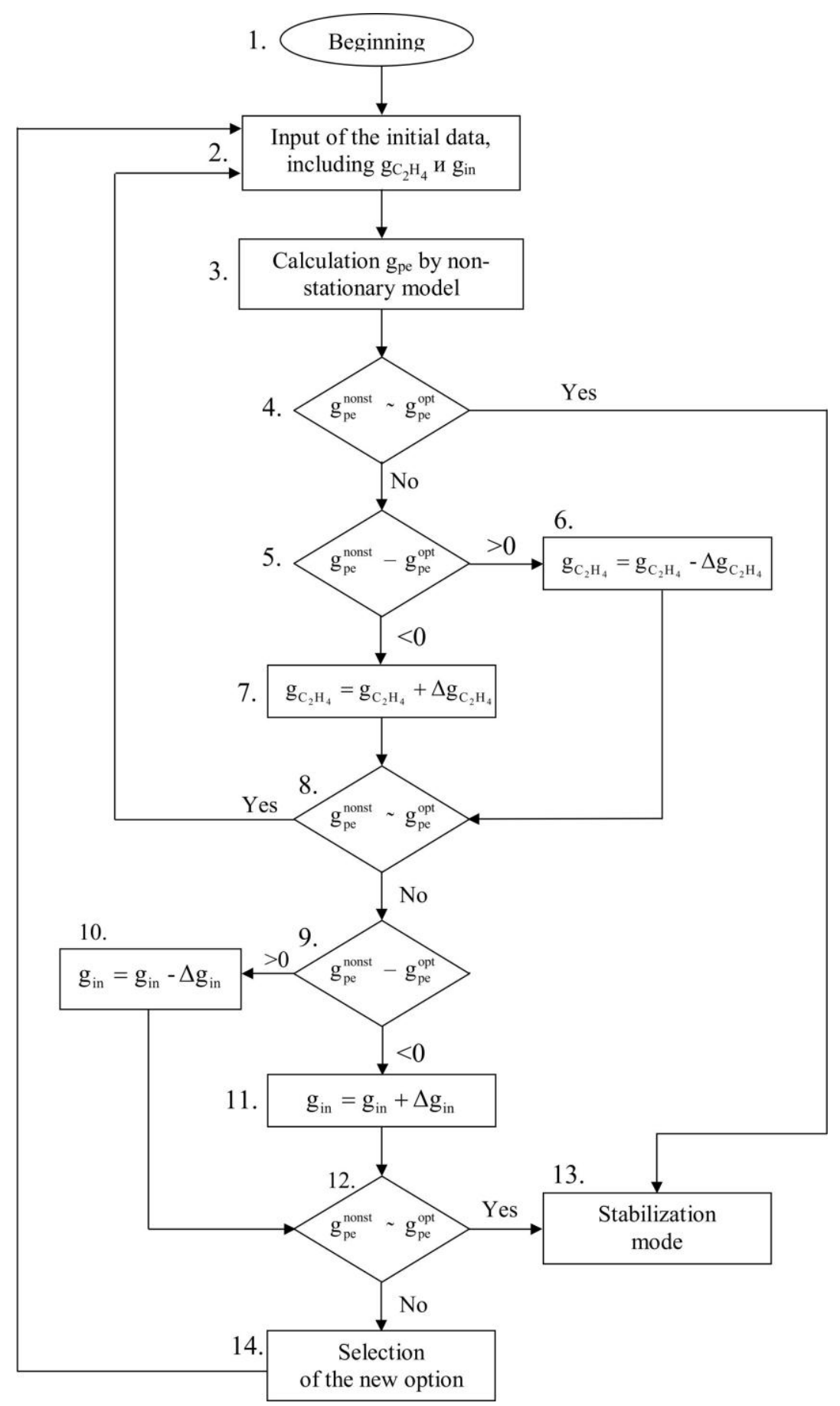


7. Results of calculation

In case productivities of polyethylene don't agree with its stationary value the process is regulated by change of value $\theta$ on the account of change in turn of amount of ethylene $g_{\mathrm{C}_{2} \mathrm{H}_{4}}$ and initiator $g_{\text {in }}$, passed into polymerizator.

\section{Direct hydration of ethylene [4]}

1. Scheme of forming ethyl alcohol

$$
\mathrm{CH}_{2}=\mathrm{CH}_{2}+\mathrm{H}_{2} \mathrm{O} \leftrightarrow \mathrm{CH}_{3}-\mathrm{CH}_{2} \mathrm{OH}
$$

2. Stationary kinetic model of the process $\frac{\mathrm{d} n}{\mathrm{~d} t}=\frac{k^{\prime}}{\left(\frac{n_{\mathrm{H}_{2} \mathrm{O}}^{0}-n}{n_{\mathrm{C}_{2} \mathrm{H}_{4}}^{0}+n_{\mathrm{H}_{2} \mathrm{O}}^{0}-n} P\right)^{\frac{1}{2}}} \cdot\left(\frac{n_{\mathrm{C}_{2} \mathrm{H}_{4}}^{0}-n}{n_{\mathrm{C}_{2} \mathrm{H}_{4}}^{0}+n_{\mathrm{H}_{2} \mathrm{O}}^{0}-n} P-\right.$

$$
\left.-K_{\mathrm{eq}}^{-1} \frac{n}{n_{\mathrm{H}_{2} \mathrm{O}}^{0}-n}\right) \cdot \frac{\left(n_{\mathrm{C}_{2} \mathrm{H}_{4}}^{0}+n_{\mathrm{H}_{2} \mathrm{O}}^{0}-n\right)^{2}}{P\left(n_{\mathrm{C}_{2} \mathrm{H}_{4}}^{0}+n_{\mathrm{H}_{2} \mathrm{O}}^{0}\right)}
$$

3. Equation of heat balance and pressure loss:

$$
\begin{gathered}
\frac{4}{\rho_{\text {cat }} \pi D^{2}} \frac{\mathrm{d} T}{\mathrm{~d} l}=-\frac{\sum_{i=1} r \Delta H_{\mathrm{R}}}{\sum_{i}^{3} n_{i} C_{p i}}-Q_{\mathrm{h}}, \\
\frac{\mathrm{d} P}{\mathrm{~d} l}=-\left(\frac{150}{\operatorname{Re}}+1.75\right) \frac{\rho_{\mathrm{gas}} u_{0}^{2}(1-\varepsilon)}{d_{\mathrm{p}} \mathrm{g} \varepsilon^{3}} \cdot 0.987 \cdot 10^{-5} .
\end{gathered}
$$

3. Function of operation:

1) $\theta=\frac{G_{\text {cur }}}{G_{\text {init }}} \quad$ (without additions of phosphorus acid);
$\theta^{\prime}=\frac{G_{\mathrm{cur}}+G_{\mathrm{add}}}{G_{\text {init }}^{\prime}}=\frac{G_{\mathrm{cur}}^{\prime}}{G_{\text {init }}^{\prime}}$ (with additions of phosphorus acid)

4. Dynamic kinetic model:

$$
\begin{aligned}
& \frac{\mathrm{d} n}{\mathrm{~d} t}=\frac{k^{\prime} \cdot \theta}{\left(\frac{n_{\mathrm{H}_{2} \mathrm{O}}^{0}-n}{n_{\mathrm{C}_{2} \mathrm{H}_{4}}^{0}+n_{\mathrm{H}_{2} \mathrm{O}}^{0}-n} P\right)^{\frac{1}{2}}} \cdot\left(\frac{n_{\mathrm{C}_{2} \mathrm{H}_{4}}^{0}-n}{n_{\mathrm{C}_{2} \mathrm{H}_{4}}^{0}+n_{\mathrm{H}_{2} \mathrm{O}}^{0}-n} P-K_{\mathrm{eq}^{-q}}^{-1} \frac{n}{n_{\mathrm{H}_{2} \mathrm{O}}^{0}-n}\right) \times \\
& \times \frac{\left(n_{\mathrm{C}_{2} \mathrm{H}_{4}}^{0}+n_{\mathrm{H}_{2} \mathrm{O}}^{0}-n\right)^{2}}{P\left(n_{\mathrm{C}_{2} \mathrm{H}_{4}}^{0}+n_{\mathrm{H}_{2} \mathrm{O}}^{0}\right)} .
\end{aligned}
$$

6. Regressive dependence of the current value of phosphorus acid on time:

$$
y=G_{\text {cur }}=f(\tau)=0.001 \tau^{2}-2.328 \tau+6543 .
$$

The system of equations (23), (17), (20) with account of (21-24) is a complete nonstationary mathematical model.

Output of ethyl alcohol is maintained at stationary level with loss expenditures for consumption of phosphorus acid (291.6 in proposed method against 637.5 - in industrial one).

As the results of operating the processes $\mathrm{N} \mathrm{1,} 2$ and 3, the use of own function of non-

\begin{tabular}{|c|c|c|c|c|c|c|c|}
\hline \multicolumn{5}{|c|}{ Industrial way } & \multicolumn{3}{|c|}{ Proposed way } \\
\hline $\begin{array}{c}\text { Time } \\
\text { breaks of } \\
\text { catalyst } \\
\text { work }\end{array}$ & $\begin{array}{c}\text { Rate of } \\
\text { removing } \\
\text { phos. } \\
\text { acid } \mathrm{kg} / \mathrm{h}\end{array}$ & $\begin{array}{l}\text { Amount of } \\
\text { removed phos. } \\
\text { acid for every } \\
50 \text { hours, kg }\end{array}$ & $\begin{array}{c}\text { Current } \\
\text { amount of } \\
\text { phos. } \\
\text { acid } \mathrm{G}_{\mathrm{cur}}, \mathrm{kg}\end{array}$ & $\begin{array}{l}\text { Control function } \\
\text { (without additions) } \\
\qquad \theta=\frac{G_{\text {cur }}}{G_{\text {init }}}\end{array}$ & $\begin{array}{l}\text { Additions } \\
\text { of } \\
\text { phos.acid } \\
G_{\text {add }}, \mathrm{kg}\end{array}$ & $\begin{array}{c}\begin{array}{c}\text { Current amount of } \\
\text { phos.acid }\end{array} \\
G_{\text {cur }}^{1}=G \text { cur }+ \text { Gadd }, \mathrm{kg}\end{array}$ & $\begin{array}{l}\text { Control function } \\
\text { (with additions) } \\
\theta=G_{\text {cur }}^{1} / G_{\text {init }}^{1}\end{array}$ \\
\hline 0 & 0 & 0 & 6534 & 1 & - & $\begin{array}{llll}- & & & \\
-\end{array}$ & - \\
\hline $0-50$ & 2.00 & 100 & 6434 & 0.9847 & - & - & 0.985 \\
\hline $50-100$ & 1.95 & 97.5 & 6336.5 & 0.9698 & 66.8 & 6403.3 & 0.98 \\
\hline $100-150$ & 1.9 & 95.0 & 6241.5 & 0.9552 & $\begin{array}{llll}- & \\
\end{array}$ & $\begin{array}{llll}- & \\
\end{array}$ & 0.98 \\
\hline $150-200$ & 1.8 & 90 & 6151.5 & 0.9415 & 58.3 & 6209.8 & 0.961 \\
\hline $200-250$ & 1.7 & 85 & 6066.5 & 0.9285 & - & - & 0.98 \\
\hline $250-300$ & 1.4 & 70 & 5996.5 & 0.9177 & 89.1 & 6085.6 & 0.966 \\
\hline $300-350$ & 1.2 & 60 & 5936.5 & 0.9086 & - & - & 0.98 \\
\hline $350-400$ & 1.0 & 50 & 5886.5 & 0.9009 & 77.4 & 5963.9 & 0.967 \\
\hline $400-450$ & 0.8 & 40 & 5846.5 & 0.8947 & - & - & 0.98 \\
\hline $450-500$ & 0.2 & 10 & 5836.5 & 0.8932 & - & - & 0.98 \\
\hline & & $\Sigma 697.5$ & & & $\Sigma 291.6$ & & \\
\hline
\end{tabular}
stationarity chosen for every process, permitted to preserve productivities of the purpose products at optimum, stationary level found out at optimization of entire CTC.

7. Results of calculation:

Table 2. Comparison of results of calculating hydration process in industrial and proposed ways of its implementation 


\section{Oxidative conversion of ethyl alcohol into vinegar acid [5]}

1. Stoichiometric scheme of the process

$$
\begin{aligned}
& \mathrm{C}_{2} \mathrm{H}_{5} \mathrm{OH}+\frac{1}{2} \mathrm{O}_{2} \stackrel{k_{1}}{\longrightarrow} \mathrm{CH}_{3} \mathrm{CHO}+\mathrm{H}_{2} \mathrm{O} \\
& \mathrm{CH}_{3} \mathrm{CHO}+\frac{1}{2} \mathrm{O}_{2} \stackrel{k_{2}}{\longrightarrow} \mathrm{CH}_{3} \mathrm{COOH} \\
& \mathrm{C}_{2} \mathrm{H}_{5} \mathrm{OH}+\mathrm{CH}_{3} \mathrm{COOH} \stackrel{k_{3}}{\longrightarrow} \mathrm{CH}_{3} \mathrm{COOC}_{2} \mathrm{H}_{5}+\mathrm{H}_{2} \mathrm{O} \\
& \mathrm{CH}_{3} \mathrm{CHO}+2 \frac{1}{2} \mathrm{O}_{2} \stackrel{k_{4}}{\longrightarrow} 2 \mathrm{CO}_{2}+2 \mathrm{H}_{2} \mathrm{O}
\end{aligned}
$$

2. Stationary kinetic model of the process:

$$
\begin{aligned}
& \frac{N_{1}^{0}}{\rho_{\text {cat }} \cdot \frac{\pi D^{2}}{4}} \frac{\mathrm{dA}_{1}}{\mathrm{~d} l}=\frac{k_{3} b_{1} b_{3} P_{1} P_{4}}{N_{1}^{0}\left(1+b_{1} P_{1}+b_{2} \sqrt{P_{2}}+b_{3} P_{4}+b_{4} P_{5}\right)^{2}} \\
& \frac{N_{1}^{0}}{\rho_{\text {cat }} \cdot \frac{\pi D^{2}}{4}} \frac{\mathrm{dA}_{2}}{\mathrm{~d} l}=\frac{k_{2} b_{2} \sqrt{P_{2}} b_{4} P-k_{3} b_{1} b_{3} P_{1} P_{4}}{N_{1}^{0}\left(1+b_{1} P_{1}+b_{2} \sqrt{P_{2}}+b_{3} P_{4}+b_{4} P_{5}\right)^{2}} \\
& \frac{N_{1}^{0}}{\rho_{\text {cat }} \cdot \frac{\pi D^{2}}{4}} \frac{\mathrm{dA}}{\mathrm{d} l}=\frac{k_{1} b_{1} b_{2} P_{1} \sqrt{P_{2}}-k_{2} b_{2} \sqrt{P_{2}} b_{4} P_{5}-k_{4} b_{4} P_{5} P_{2}\left(1+b_{1} P_{1}+b_{2} \sqrt{P_{2}}+b_{3} P_{4}+b_{4} P_{5}\right)}{N_{1}^{0}\left(1+b_{1} P_{1}+b_{2} \sqrt{P_{2}}+b_{3} P_{4}+b_{4} P_{5}\right)^{2}} \\
& \frac{N_{1}^{0}}{\rho_{\text {cat }} \cdot \frac{\pi D^{2}}{4}} \frac{2 k_{4} P_{2} b_{4} P_{5}}{\mathrm{~d} l}=\frac{\mathrm{d} A_{4}}{N_{1}^{0}\left(1+b_{1} P_{1}+b_{2} \sqrt{P_{2}}+b_{3} P_{4}+b_{4} P_{5}\right)}
\end{aligned}
$$

3. Equation of heat balance and pressure bosses:

$$
\begin{aligned}
& \frac{N_{1}^{0}}{\rho_{\mathrm{k}} \cdot \frac{\pi D^{2}}{4}} \frac{\mathrm{d} T}{\mathrm{~d} l}=-\frac{\sum_{j=1}^{4} r_{j} \Delta H_{R j}}{\sum_{i=1}^{8} n_{i} C_{p i}}-\frac{\alpha\left(T-T_{r}\right)}{\sum_{\mathrm{i}=1}^{8} n_{\mathrm{i}} C_{p i}}, \\
& \frac{\mathrm{d} P}{\mathrm{~d} l}=-\left(\frac{150}{\operatorname{Re}}+1.75\right) \cdot \frac{\rho_{\mathrm{gas}} u_{0}^{2}(1-\varepsilon)}{d_{\mathrm{p}} g \varepsilon^{3}} \cdot 0.987 \cdot 10^{-5}
\end{aligned}
$$

\section{Etherification of vinegar acid by ethyl alcohol [5]:}

1. Stoichiometric scheme of the process;

$$
\mathrm{C}_{2} \mathrm{H}_{5} \mathrm{OH}+\mathrm{CH}_{3} \mathrm{COOH} \rightarrow \mathrm{CH}_{3} \mathrm{COOC}_{2} \mathrm{H}_{5}+\mathrm{H}_{2} \mathrm{O}
$$

2. Stationary kinetic model of the process;

$$
\frac{N_{1}^{0}}{\rho_{\text {cat }} \cdot \frac{\pi D^{2}}{4}} \frac{\mathrm{d} X}{\mathrm{~d} l}=\frac{k b_{1} b_{2} P^{2}(1-X)(\varphi-X)}{N_{1}^{0}\left(1+b_{1} \frac{1-X}{1+\varphi} P+b_{2} \frac{\phi-X}{1+\varphi} P\right)^{2}(1+\varphi)^{2}}
$$

3. Equation of heat balance and pressure losses:

$$
\begin{aligned}
& \frac{4}{\rho_{\mathrm{cat}} \pi D^{2}} \frac{\mathrm{d} T}{\mathrm{~d} l}=-\frac{\Delta H_{\mathrm{R}} \mathrm{N}_{1}^{0} \frac{4}{\rho_{\mathrm{cat}} \pi D^{2}} \frac{\mathrm{d} x}{\mathrm{~d} l}+\alpha\left(T-T_{\mathrm{r}}\right)}{\left(C_{p 1} n_{1}+C_{\mathrm{p} 4} n_{4}+C_{\mathrm{p} 3} n_{3}+C_{\mathrm{p} 7} n_{7}\right)} \\
& \frac{\mathrm{d} P}{\mathrm{~d} l}=-\left(\frac{150}{\operatorname{Re}}+1.75\right) \frac{\rho_{\mathrm{gas}} u_{0}^{2}(1-\varepsilon)}{d_{\mathrm{p}} g \varepsilon^{3}} 0.987 \cdot 10^{-5}
\end{aligned}
$$


Results of calculation the optimum out pus have been found out on the purpose products of two letter processes. As the processes of oxidative conversion of ethyl alcohol into vinegar acid and esterification of vinegar acid by ethyl alcohol within 50 hours of a catalysts work of clinoptilolite and $\beta$-zeolite display stable activity, then at calculation of region there were used mathematical models cited for stationary conditions.

\section{Conclusion}

The proposed in the work list of main actions which should be fulfilled for preserving stability of work of chemico-technological system, way serve as a guide for calculation of any region of CTC, including the processes with possible non-stationarity of their proceeding.

\section{References}

1. Aliyev A.M., Osmanova I.I., Mammadov E.M.,
2. Safarov A.R., Huseynova A.M. Development of kinetic models of non-stationary processes with consideration for characteristic features of changing catalyst activity. Chem. Ind. St. Petersburg. 2013. V. 95. No 2. P. 64-75.

3. Aliyev A.M., Osmanova I.I., Safarov A.R., Huseynova A.M., Yariev V.M. Equation of the processes of ethane pyrolysis in non-stationary conditions. Azerb. Chem. Journ. 2017. No 2. P. 10-15.

4. Aliyev A.M., Osmanova I.I., Safarov A.R., Huseynova A.M. Development of non-stationary mathematical model of the processes of ethylene polymerization. Azerb. Chem. Journ. 2017. No 3. P. 20-23.

5. Aliyev A.M., Osmanova I.I., Safarov A.R., Huseynova A.M., Ismaylov A.Q. Control of the processes of direct ethylene hydration with consideration for dynamics of its proceeding. Azerb. Chem. Journ. 2017. No 4. P. 17-24.

6. Safarov A.R. Modeling and optimization of the processes of obtaining vinegar acid and ethylacetate by combined technology. Synopsis of dis. cand. of techn. sci. Institute of Chemical Problems. ANAS, Baku, 2006. 26 p.

\section{РЕШЕНИЕ ВОПРОСОВ НЕСТАЦИОНАРНОСТИ ПРОЦЕССОВ ЭТИЛЕНОВОГО РЕГИОНА ХИМИКО-ТЕХНОЛОГИЧЕСКОГО КОМПЛЕКСА ПО ПЕРЕРАБОТКЕ ГАЗОВ КРЕКИНГА И ПИРОЛИЗА}

\section{И.И.Османова}

Изучена динамика процессов самого большого - этиленового региона ХТК, выбраны функции, учитывающие нестационарность процессов, составлены их динамические кинетические модели и на основе полных математических описаний решены задачи их оптимального управления, что в итоге позволило выполнить поставленную цель - сохранить оптимальные производительности целевых продуктов, найденных при оптимизации ХТК для стационарных условий их протекания.

Ключевые слова: нестациионарность, функиия дезактивации, управление, производительность, динамика, активность катализатора, блок схема.

\section{KREKINQ Və PİROLIZ QAZLARININ EMALININ KIMYOVI TEXNOLOJI KOMPLEKSININ ETILEN

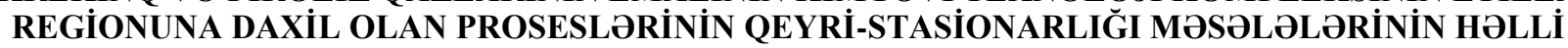 \\ İ.İ.Osmanova}

KTK-in ən böyük - etilen regionu proseslərinin dinamikası öyrənilib, proseslərin qeyri-stasionarlığını nəzərə alan funksiyalar seçilib, onların dinamik kinetik modelləri tərtib və tam riyazi təsvir əsasında onların optimal idarə edilməsi məsələsi həll olunub ki, bu da nəticədə qarşıya qoyulmuş məqsədi - məqsədli məhsulların KTK-in stasionar şəraitdə optimallaşdırılması zamanı alınan məhsuldarlığını sabit saxlamaq məsələsini həll etməyə imkan verdi.

Açar sözləri: qeyri-stasionarlıq, deaktivasiya funksiyası, idarəetmə, məhsuldarlıq, dinamika, katalizatorun aktivliyi, blok-sxem.

Additional remarks for references

1. $d_{\text {ins }}$-inside diameter of tube, $\mathrm{m}$;

2. $d_{\text {ins }}^{f}$ - diameter in the end of time's interval;

3 . $E_{\text {in }}$ - energy of activation of initiator's decomposition, $\mathrm{kJ} / \mathrm{mol}$;

4. $g_{\mathrm{C}_{2} \mathrm{H}_{4}}^{\exp }, g_{\mathrm{C}_{2} \mathrm{H}_{4}}^{\text {cal }}-$ experimental and calculated yield of ethylene, $\mathrm{kg} / \mathrm{h}$;

5. $g_{\text {in }}$ - current amount of initiator, $\mathrm{kmol} / \mathrm{h}$;

6. $g_{\mathrm{pe}}^{\text {nonst }}, g_{\mathrm{pe}}^{\mathrm{opt}}-$ non-stationary and optimal amounts of polyethylene, $\mathrm{kmol} / \mathrm{h}$;
7. $G_{\text {cur }}, G_{\text {init }}$ - current and initial amounts of phosphorus acid, $\mathrm{kg}$;

8. $G_{\text {add }}$-additions of phosphorus acid, $\mathrm{kg}$;

9. $P_{f}$ - pressure at outlet from reactor, atm;

10. $Q_{h}$ - warmth of heating of ethylene, $\mathrm{kkal} / \mathrm{mol}$;

11. $T_{\mathrm{r}}$ - temperature of refrigerant, $\mathrm{K}$;

12. $Y_{\text {cal }}, \bar{Y}_{\text {cal }}$ - regressive dependence of ethylene yield;

13. $\rho_{\text {cat }}, \rho_{\text {gas }}-$ densities of catalizator and gas, $\mathrm{kg} / \mathrm{m}^{3}$;

14. $K_{\text {eq }}-$ constant of equilibrium. 